\title{
8. X-RAY MINERALOGY OF DEEP SEA DRILLING PROJECT LEGS 51 THROUGH 53, WESTERN NORTH ATLANTIC
}

\author{
Ulrich Mann and German Müller, Institut für Sedimentforschung, Universität Heidelberg, Heidelberg, West Germany
}

\section{INTRODUCTION}

A series of 140 samples from Holes 417A, 417B, 418A, and $418 \mathrm{~B}$ were examined semiquantitatively for their X-ray mineralogy. We selected most samples from the dominant lithologies rather than by coring the same sedimentary sequence at Sites 417 and 418 , only $8 \mathrm{~km}$ apart (Figure 1). Both sites are located at the foot of the Bermuda Rise (Figure 2) along the $M 0$ magnetic anomaly on crust of lower Aptian age. Sediments are mainly abyssal pelagic clays, with various authigenic minerals low in carbonates.

\section{METHODS}

\section{Analysis Conditions}

The mineralogical data in Tables 1 and 2 are for samples dried at $70^{\circ} \mathrm{C}$, powdered and X-rayed under the following conditions: $\mathrm{CuK} \propto$ radiation, nickel filter, $36 \mathrm{kv} / 24 \mathrm{~mA}$, and $1^{\circ}$ detection slit. Goniometer speed was $0.5^{\circ}=2 \theta / \mathrm{min}$, paper speed $10 \mathrm{~mm} / \mathrm{min}$. All samples were X-rayed from $3^{\circ}$ to $40^{\circ}=2 \theta$.

Clay minerals were identified on oriented glass smear mounts. To obtain the optimal texture, the $<2 \mu \mathrm{m}$ fraction was first disintegrated with a KLN 582 ultrasonic generator and then centrifuged to clearness. The residue was mixed to a homogeneous paste and finally placed on the glass slide. It was X-rayed in an untreated state and glycolated, when necessary, after heating.

\section{Factor Analysis}

For the bulk mineralogy, the factors used are those listed in Table 3. When multiplied by their peak heights, they add up to 100 per cent. Because of the large amount of amorphous material - i.e., volcanic glass, opal, and amorphous clay minerals - and without using the monochromator, diffuse scattering was relatively high. Thus, if these components vary, the factors have to be redetermined. These factors can only be used for sedimentary types that are very similar to those above; for example, they cannot be applied to nearshore sediments on account of their higher crystallinity.

For the clay mineralogy, we used the method discussed by Biscaye (1965). The factors in Table 4 were multiplied by the peak area, determined planimetrically; the sum of these was 100 per cent. The kaolinite/chlorite peak at $12.3 \AA$ $=2 \theta$ was divided by peak splitting at 24 to $25^{\circ}=2 \theta-$ a factor obtained from the ratio:

$$
\frac{3.58 \AA \text { peak area }}{3.58 \AA+3.54 \AA \text { peak area }}
$$

The carbonate contents were determined gasometrically by carbonate bomb (Müller and Gastner, 1971). X-ray numerical data for all holes are presented in tabular form on the core barrel sheets following the site report chapters. Mineral identifications were made manually and peak areas measured with the aid of a template having graduated triangles. The amount of amorphous material was not determined quantitatively.

\section{RESULTS}

X-ray results are listed in Tables 1 and 2. A graphic representation in Figures 3 through 5 places the samples at their proper stratigraphic depths.

Unit 1 and 2 pelagic clays are characterized mainly by a chlorite component in the dominant clay universal spectrum. Rhodochrosite and dolomite appear in the base of Unit 2 above the first clinoptilolite occurrences in Unit 1. Palygorskite occurs in the nonzeolitic interval of midEocene radiolarian clays. Multicolored Cretaceous clays are rich in clinoptilolite and cristobalite from former radiolarian tests.

Palygorskite increases in abundance downhole in the Upper Cretaceous. In the Middle Cretaceous black clay facies, the clay-mineral assemblage changes to dominantly mixed-layer varieties; illite peaks are asymmetric; zeolites plus cristobalites disappear; and quartz (and, periodically, calcite) becomes an important constituent. Several authigenic phases were detected, including pyrite, gypsum, barite, and apatite (phosphates).

\section{REFERENCES}

Biscaye, P. E., 1965. Mineralogy and sedimentation of recent deep-sea clay in the Atlantic Ocean and adjacent seas and oceans, Geol. Soc. Am. Bull., v.76, no. 7, p. 803-832.

Müller, G. and Gastner, M., 1971. The "Carbonate Bomb," a simple device for the determination of the carbonate content in sediments, soils, and other materials, $N$. Jb. Mineral., v. 10, p. $466-469$. 
U. MANN, G. MÜLLER

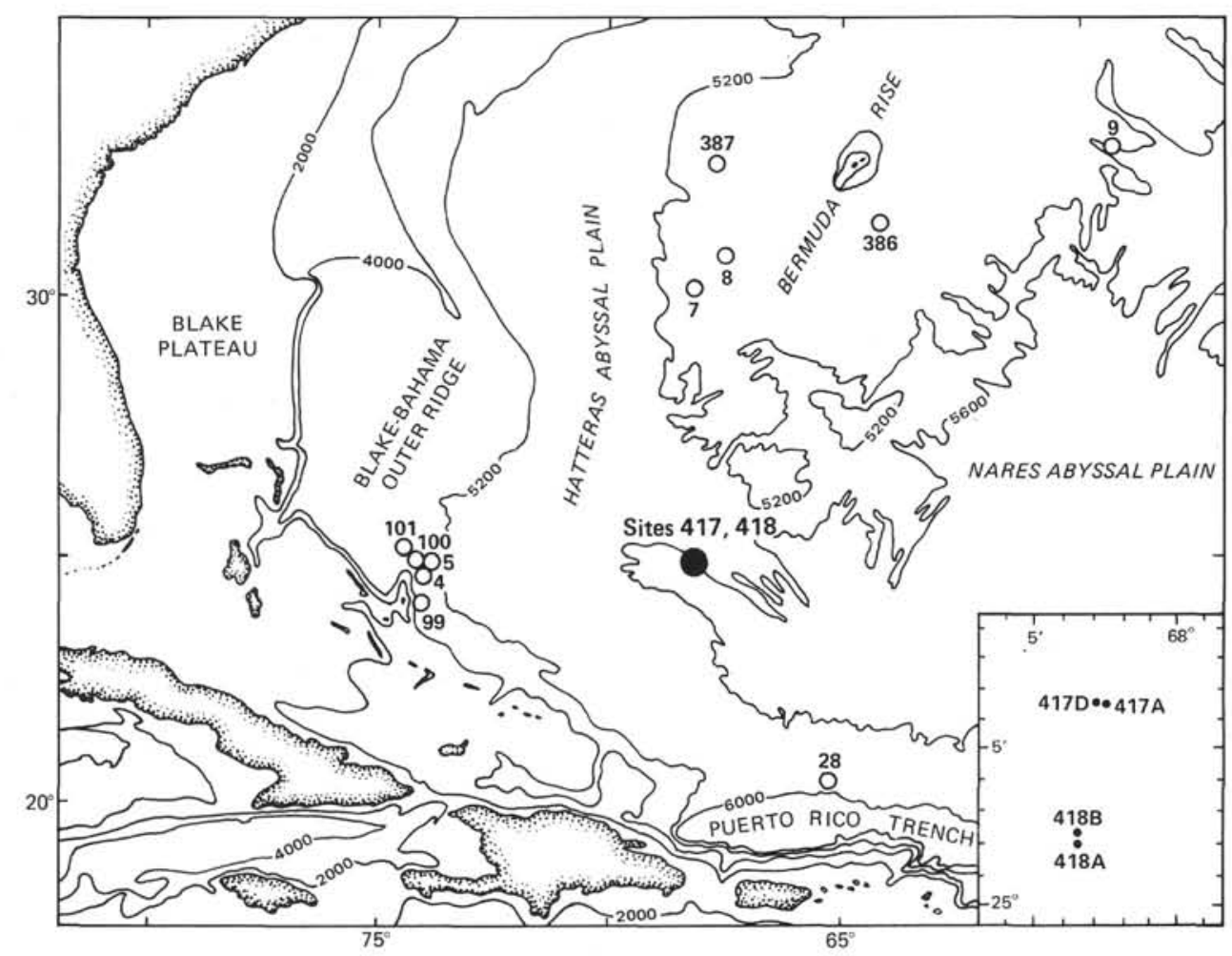

Figure 1. Location of Sites 417 and 418. 
$418 \mathrm{~B}$

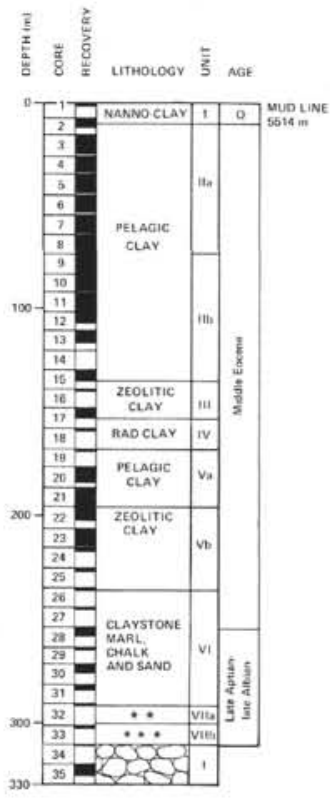

4184

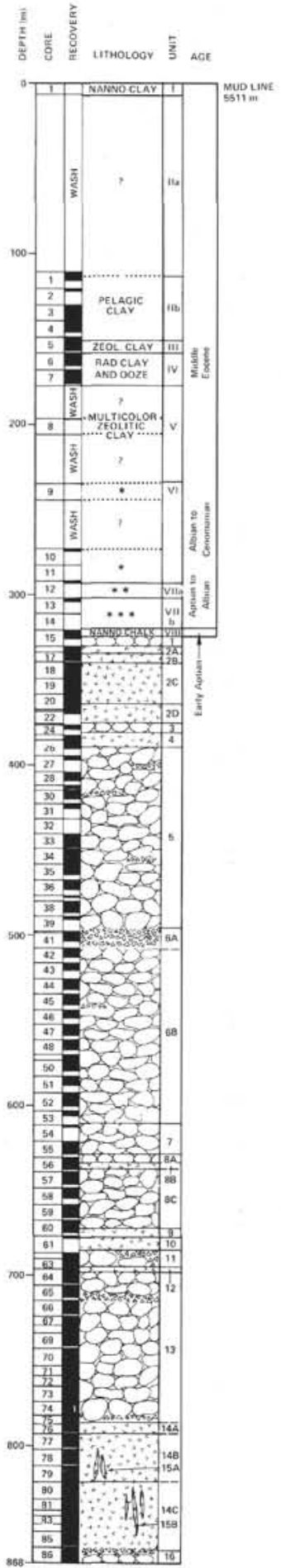

4170

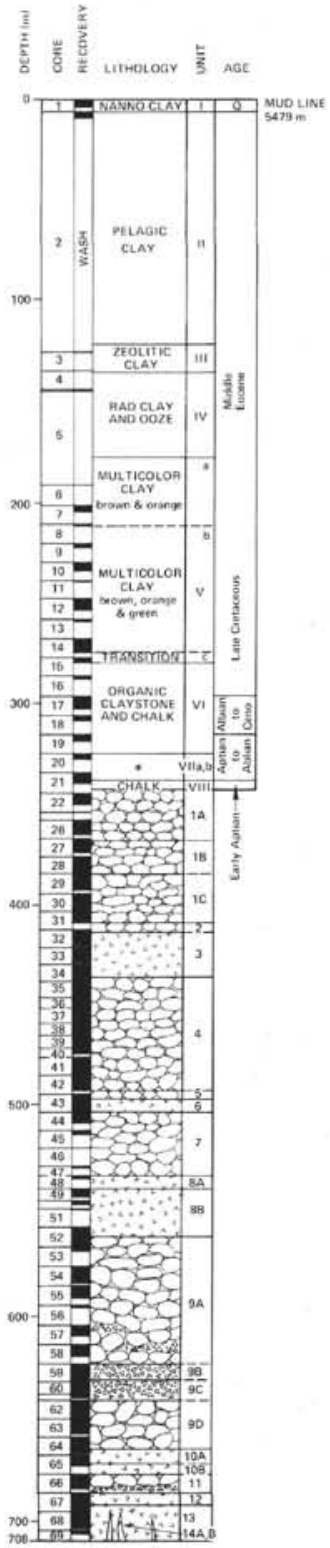

$417 \mathrm{~A}$

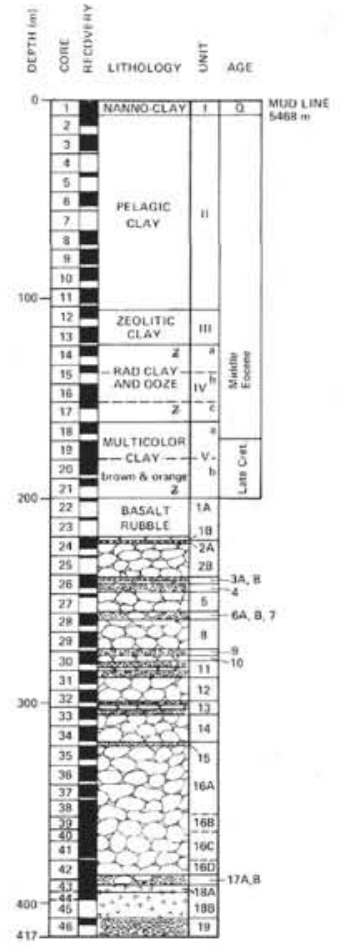

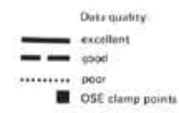

Figure 2. Stratigraphy of sediment and basement sections of Sites 417 and 418 . 
TABLE 1

X-Ray Mineralogical Analysis, Site 417, Holes 417A and 417D

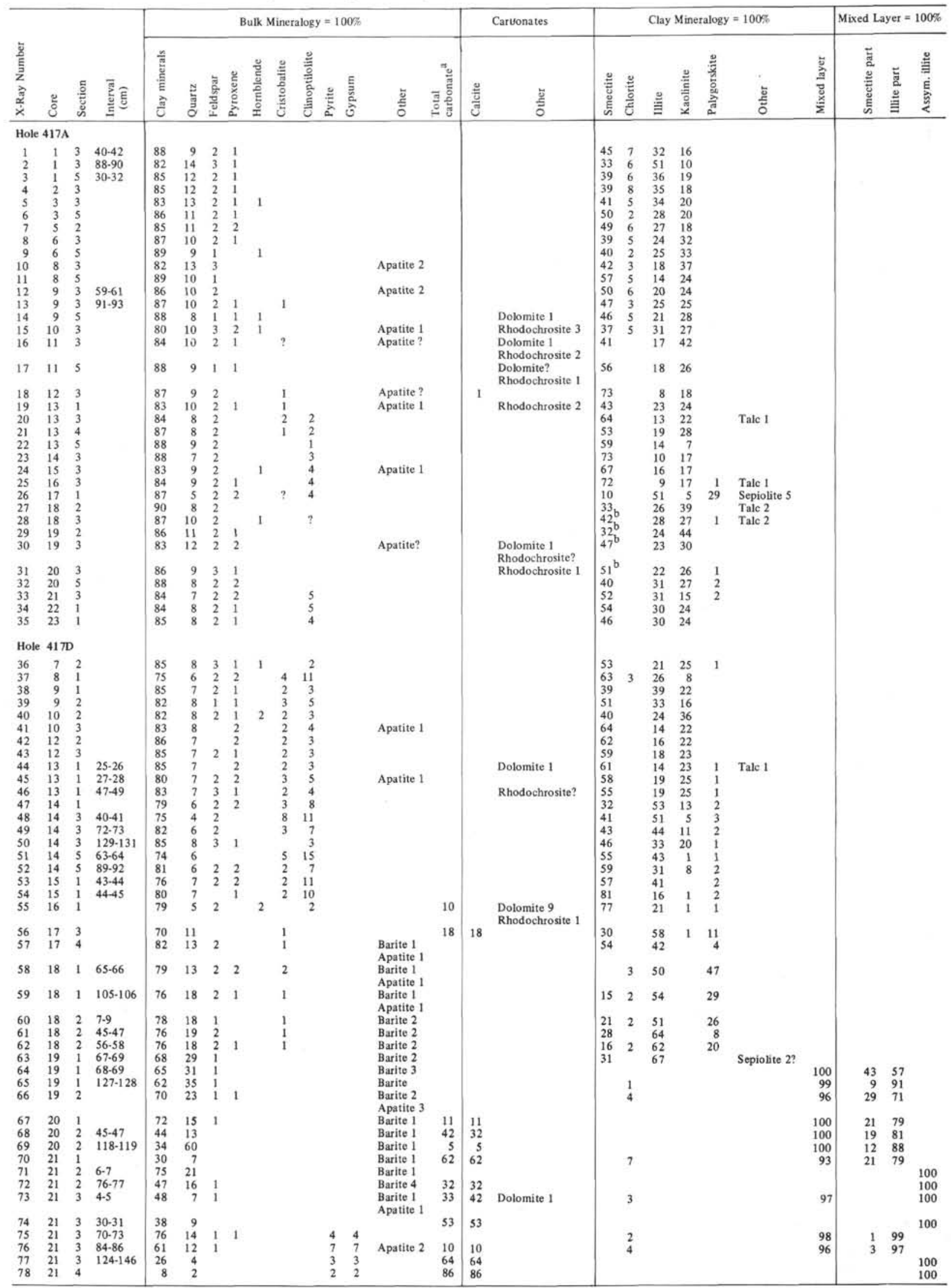

${ }^{\mathrm{a}}$ If $>3$ per cent.

Smeetite with shoulders, 
TABLE 2

X-Ray Mineralogical Analysis, Site 418, Holes 418A and 418B

\begin{tabular}{|c|c|c|c|c|c|c|c|c|c|c|c|c|c|c|c|c|c|c|c|c|c|c|c|c|c|c|}
\hline \multirow[b]{2}{*}{ 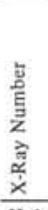 } & \multirow[b]{2}{*}{ ¿ } & \multirow[b]{2}{*}{ 气ू } & \multirow[b]{2}{*}{ 总言 } & & & & Bull & $\mathrm{k}$ Mir & neralo & $y=1$ & $00 \%$ & & & & & Carbonates & & & Clay N & ineralog & $z y=1$ & $00 \%$ & & Mixed Lay & yer $=$ & \\
\hline & & & & $\begin{array}{l}\text { है } \\
\text { है } \\
\text { 音 } \\
\text { 音 }\end{array}$ & 칠 & 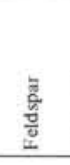 & 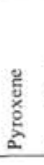 & 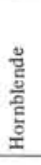 & 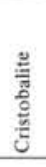 & 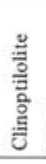 & 产 & 麇 & 衣 & 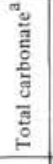 & $\frac{\mathscr{n}}{\pi}$ & 总 & 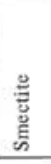 & $\begin{array}{l}\frac{\dddot{\Xi}}{0} \\
\text { है }\end{array}$ & 总 & 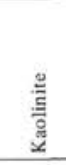 & 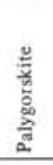 & 气ั & 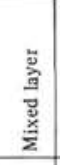 & 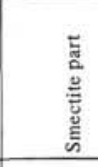 & $\begin{array}{l}\text { 폴. } \\
\text { 兰 } \\
\text { 总 }\end{array}$ & 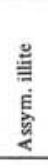 \\
\hline Hole & $418 \mathrm{~A}$ & & & & & & & & & & & & & & & & & & & & & & & & & \\
\hline 1 & 1 & 1 & $65-67$ & 77 & 14 & $4 \mathrm{P} / 2 \mathrm{~K}$ & 1 & & & & & & Apatite 1 & & & Dolomite 1 & 27 & 10 & 52 & 11 & & & & & & \\
\hline 2 & 1 & 4 & $60-62$ & 79 & 13 & $4 \mathrm{P} / 2 \mathrm{~K}$ & 1 & & & & & & & & & Dolomite I & 25 & 9 & 56 & 10 & & & & & & \\
\hline 3 & 1 & 2 & $83-85$ & 88 & 9 & 2 & 1 & & & & & & & & & & 57 & & 21 & 22 & & & & & & \\
\hline 4 & 2 & 1 & $14-16$ & 87 & 10 & 2 & 1 & & & & & & & & & & 49 & & 28 & 23 & & & & & & \\
\hline 5 & 3 & 2 & $35-36$ & 85 & 10 & 3 & 1 & 1 & & & & & & & & & 51 & & 22 & 27 & & & & & & \\
\hline 6 & 3 & 4 & $54-56$ & 86 & 9 & 2 & $i$ & i & & & & & & & & Rhodochrosite 1 & 50 & & 21 & 29 & & & & & & \\
\hline 7 & 3 & 6 & $11-13$ & 75 & 9 & $5 \mathrm{P} / 7 \mathrm{~K}$ & & & 2 & & & & Apatite 1 & & & Rhodochrosite I & 62. & & 15 & 23 & & & & & & \\
\hline 8 & 3 & 6 & $44-46$ & 84 & 10 & 2 & & & & & & & Apatite 1 & & & Rhodochrosite 2 & $43^{b}$ & 25 & & & & & & & & \\
\hline 9 & 1 & 1 & $69-71$ & 86 & 11 & 2 & & & & & & & & & & Rhodochrosite 1 & $45^{b}$ & 23 & 32 & & & & & & & \\
\hline 10 & 4 & 5 & $47-49$ & 87 & 10 & 2 & & & 1 & & & & & & & & 40 & 29 & 31 & & & & & & & \\
\hline 11 & 5 & 1 & 18.20 & 87 & 9 & 2 & & & 1 & & & & & & & Rhodochrosite 1 & 43 & 22 & 33 & & & Sepiolite 2 & & & & \\
\hline 12 & 5 & 3 & $67-69$ & 88 & 9 & 2 & & & i & & & & & & & & 62 & 13 & 25 & & & & & & & \\
\hline 13 & 5 & 4 & $28-30$ & 85 & 9 & 2 & 1 & 1 & 1 & & & & & & & Rhodochrosite 1 & 63 & 17 & 20 & & & & & & & \\
\hline 14 & 5 & 4 & $109-111$ & 86 & 9 & 2 & $i$ & 1 & 1 & & & & & & & & 54 & 20 & 26 & & & & & & & \\
\hline 15 & 6 & 1 & 67.70 & 85 & 7 & 2 & & & 2 & 4 & & & & & & & 63 & 21 & 16 & & & & & & & \\
\hline 16 & 7 & 2 & $63-65$ & 84 & 5 & 2 & 1 & & 1 & 7 & & & & & & & 73 & 16 & 8 & 1 & & Talc 2 & & & & \\
\hline 17 & 7 & 4 & $28-30$ & 81 & 9 & 2 & & & 1 & 7 & & & & & & & 73 & 16 & 8 & i & & Tale 2 & & & & \\
\hline 18 & 7 & 5 & $46 \cdot 48$ & 89 & 5 & 2 & & & 2 & & & & Apatite 1 & & 1 & & 5 & & 39 & trace & 61 & Sepiolite 5 & & & & \\
\hline 19 & 8 & 1 & $144-146$ & 90 & 7 & 2 & 1 & & & & & & & & & & 61 & & 16 & 21 & 1 & Talc 1 & & & & \\
\hline 20 & 10 & 1 & $109-111$ & 71 & 10 & 2 & & & 4 & & 4 & & Barite 4 & & & & 16 & trace & 75 & & 9 & & & & & \\
\hline 21 & 12 & 1 & $69-71$ & 62 & 22 & 1 & & & 1 & & & & $\begin{array}{l}\text { Apatite } 5 \\
\text { Barite } 1\end{array}$ & 13 & 13 & & 65 & & 35 & & & & & & & \\
\hline 22 & 13 & $i$ & $62-64$ & 37 & 15 & & & & & & & & Dante i & 48 & 48 & & & & & & & & 100 & 7 & 93 & \\
\hline 23 & 13 & 2 & $15-18$ & 72 & 26 & 2 & & & & & & & & & & & & & & & & & 100 & 5 & 95 & \\
\hline 24 & 13 & 2 & $109-110$ & 73 & 24 & $i$ & & & & & & & $\begin{array}{l}\text { Barite 1 } \\
\text { Apatite 1 }\end{array}$ & & & & & & & & & & 100 & 3 & 97 & \\
\hline Hole & $418 \mathrm{~B}$ & & & & & & & & & & & & & & & & & & & & & & & & & \\
\hline 1 & 1 & 1 & $141-143$ & 60 & 17 & 5 & 2 & 2 & & & & & & 14 & 12 & Dolomite 2 & 30 & 13 & 36 & 21 & & & & & & \\
\hline 2 & 2 & 1 & $51-53$ & 74 & 20 & 4 & 2 & & & & & & & & & & 31 & 5 & 40 & 24 & & & & & & \\
\hline 3 & 3 & 1 & $67-69$ & 73 & 23 & 2 & 2 & & & & & & & & & & 36 & 6 & 39 & 19 & & & & & & \\
\hline 4 & 3 & 3 & $67-69$ & 73 & 22 & 3 & 2 & & & & & & & & & & 41 & 6 & 33 & 20 & & & & & & \\
\hline 5 & 4 & 1 & $82-84$ & 76 & 10 & 3 & 1 & & & & & & & & & & 45 & 5 & 36 & 14 & & & & & & \\
\hline 6 & 5 & 1 & $74-76$ & 77 & 19 & 3 & $i$ & & & & & & & & & & 42 & 4 & 30 & 24 & & & & & & \\
\hline 7 & 6 & 6 & $36 \cdot 38$ & 83 & 16 & 2 & 1 & & & & & & & & & & 46 & 4 & 25 & 25 & & & & & & \\
\hline 8 & 7 & 6 & $56-58$ & 81 & 16 & 2 & $i$ & & & & & & & & & & 47 & 3 & 26 & 24 & & & & & & \\
\hline 9 & 8 & 1 & 77.79 & 81 & 16 & 2 & 1 & & & & & & & & & & 42 & 4 & 23 & 31 & & & & & & \\
\hline 10 & 8 & 4 & $6-8$ & 83 & 14 & 2 & 1 & & & & & & & & & & 46 & & 17 & 27 & & & & & & \\
\hline 11 & 9 & 1 & $72-74$ & 80 & 17 & 2 & 1 & & & & & & & & & & 45 & & 28 & 27 & & & & & & \\
\hline 12 & 9 & 6 & $52-54$ & 86 & 10 & 1 & 1 & & & & & & & & & Rhodochrosite 2 & 62 & & 18 & 20 & & & & & & \\
\hline 13 & 10 & 1 & 71.73 & 86 & 12 & 2 & & & & & & & & & & & 71 & & 13 & 16 & & & & & & \\
\hline 14 & 10 & 3 & $133-134$ & 88 & 8 & 2 & & & & & & & & & & Rhodochrosite 2 & 62 & & 21 & 17 & & & & & & \\
\hline 15 & 11 & 2 & $85-87$ & 83 & 13 & 2 & 2 & & & & & & & & & & 68 & & 15 & 17 & & & & & & \\
\hline 16 & 11 & 3 & $85-87$ & 82 & 14 & 2 & 2 & & & & & & & & & & 72 & & 16 & 12 & & & & & & \\
\hline 17 & 13 & 1 & 65.67 & 77 & 19 & 2 & 2 & & & & & & & & & & 54 & & 21 & 22 & & & & & & \\
\hline 18 & 15 & 2 & $78-80$ & 81 & 15 & 2 & & 2 & & & & & & & & & 49 & & 22 & 29 & & & & & & \\
\hline 19 & 16 & 1 & $20-22$ & 87 & 12 & 1 & & & & & & & & & & & 56 & & 18 & 26 & & & & & & \\
\hline $2 \theta$ & 17 & 1 & $92-94$ & 83 & 13 & 2 & 2 & & & & & & Apatite? & & & Rhodochrosite? & 61 & & 14 & 25 & & & & & & \\
\hline 21 & 18 & 1 & $109-111$ & 80 & 11 & 2 & 2 & & 1 & 4 & & & & & & Rhodochrosite? & 64 & & 13 & 23 & & Sepiolite & & & & \\
\hline 22 & 19 & 2 & $23-25$ & 81 & 8 & 2 & 2 & & 1 & 5 & & & Apatite 1 & & & & 12 & & so & 10 & 21 & Sepiolite 6 & & & & \\
\hline 23 & 20 & 2 & $77-79$ & 83 & 12 & 2 & 2 & & & & & & Apatitie 1 & & & & 40 & & 30 & 25 & 2 & $\begin{array}{l}\text { Tale } 1 \\
\text { Talc } 3\end{array}$ & & & & \\
\hline 24 & 21 & 6 & $49-50$ & 87 & 8 & 2 & 2 & & & & & & Apatitie 1 & & & & 64 & & 12 & 20 & 1 & Talc 3 & & & & \\
\hline 25 & 22 & 1 & $22-24$ & 81 & 9 & 2 & 2 & 1 & & 5 & & & Barrite? & & & & 41 & & 31 & 24 & & Talc 4 & & & & \\
\hline 26 & 22 & 2 & $18-20$ & 76 & 13 & 2 & 2 & & & 7 & & & & & & & 43 & & 26 & 31 & & & & & & \\
\hline 27 & 23 & 1 & $15-17$ & 78 & 10 & 1 & 1 & & & 10 & & & & & & & 47 & & 31 & 22 & & & & & & \\
\hline 28 & 25 & 2 & $78-80$ & 85 & 10 & 2 & $i$ & & & 2 & & & & & & & 46 & & 28 & 26 & & & & & & \\
\hline 29 & 26 & 1 & $80-82$ & 76 & 5 & 3 & 2 & & & 5 & 3 & 4 & Apatitie 2 & & & & 95 & & 5 & & & & & & & \\
\hline 30 & 26 & 1 & $146 \cdot 148$ & 86 & 9 & 1 & 1 & & & 3 & & & & & & & 37 & & 36 & 23 & 4 & & & & & \\
\hline 31 & 27 & 1 & $5-7$ & 78 & 6 & 2 & & & 1 & 1 & & & & 12 & 12 & & 69 & & 25 & & 6 & & & & & \\
\hline 32 & 29 & 1 & $55-57$ & 78 & 7 & 1 & & & 4 & & 2 & 2 & Apatite 2 & 4 & 4 & & 60 & & 34 & & 6 & & & & & \\
\hline 33 & 30 & 2 & $84-85$ & 69 & 20 & & & & 11 & & & & $\begin{array}{l}\text { Barrite? } \\
\text { Barrite? }\end{array}$ & & & & & & & & & & 100 & 19 & 81 & \\
\hline 34 & 32 & 1 & 85.87 & 58 & 36 & & & & & & & & & 16 & 16 & & & & & & & & 100 & 2 & 98 & \\
\hline 35 & 32 & 1 & $119-121$ & 67 & 16 & 1 & & & & & & & & 16 & 16 & & & & & & & & 100 & 12 & 88 & \\
\hline
\end{tabular}

Note: $P=$ plagioclase $: K=$ potassium feldspar. 
U. MANN, G. MÜLLER

TABLE 3

Factor for Minerals Investigated, Bulk Mineralogy

\begin{tabular}{lccc}
\hline \multicolumn{1}{c}{ Mineral } & $\begin{array}{c}\text { Peak } \\
2 \theta \text { (in degrees) }\end{array}$ & $\mathrm{d}$ (in $\AA$ ) & Factor \\
\hline Quartz & 26.7 & 3.34 & 1.3 \\
Feldspar & $27.4-28.0$ & $3.25-3.18$ & 2.0 \\
Pyroxene & 29.9 & 2.99 & 2.0 \\
Hornblende & 10.5 & 8.42 & 2.0 \\
Cristobalite & $21.5-21.9$ & $4.15-4.05$ & 1.5 \\
Clinoptilolite & 9.8 & 8.99 & 2.0 \\
Pyrite & 33.0 & 2.71 & 2.0 \\
Gypsum & 11.7 & 7.56 & 2.0 \\
Rhodochrosite & 31.4 & 2.85 & 2.0 \\
Barrite & 25.9 & 3.44 & 2.0 \\
Apatite & 32.2 & 2.78 & 2.0 \\
\hline
\end{tabular}

TABLE 4

Factors for Minerals Investigated, Clay Mineralogy

\begin{tabular}{lcrc}
\hline \multicolumn{1}{c}{ Mineral } & $\begin{array}{c}\text { Peak } \\
2 \theta \text { (in degrees) }\end{array}$ & d (in $\AA$ ) & Factor \\
\hline Smectite & 5.2 & 17.0 & 1 \\
Chlorite & 12.3 & 7.2 & 2 \\
Illite & 8.8 & 10.0 & 4 \\
Kaolinite & 12.3 & 7.2 & 2 \\
Palygorskite & 8.4 & 10.5 & 1 \\
Talc & 9.4 & 9.3 & 1 \\
Sepiolite & 7.4 & 12.0 & 1 \\
Mixed layer & Same as its parts & & \\
\hline
\end{tabular}




\section{LEG 51 HOLE $417 \mathrm{~A}$}

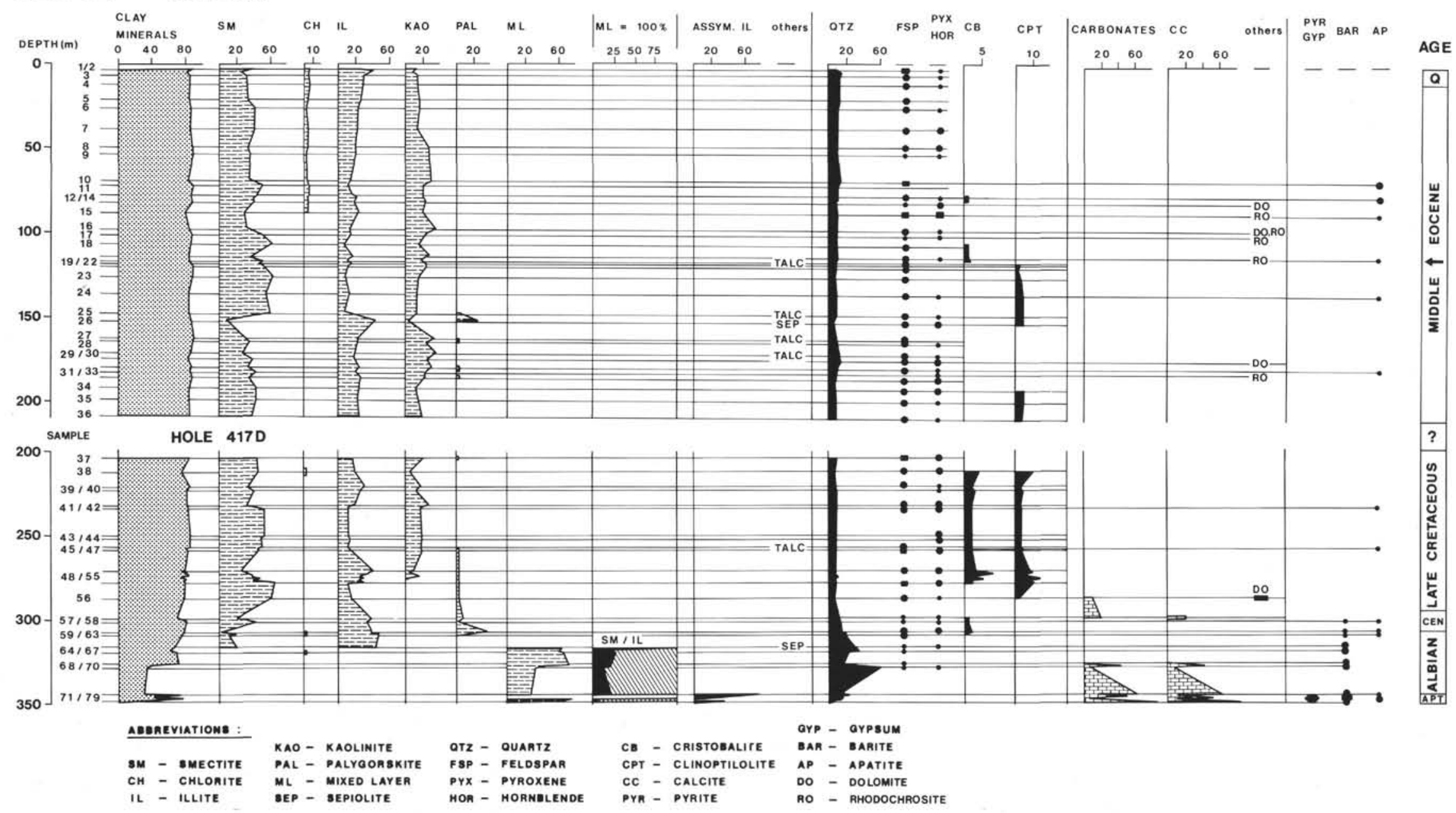

Figure 3. X-ray diffraction data, Hole $417 \mathrm{~A}$. 


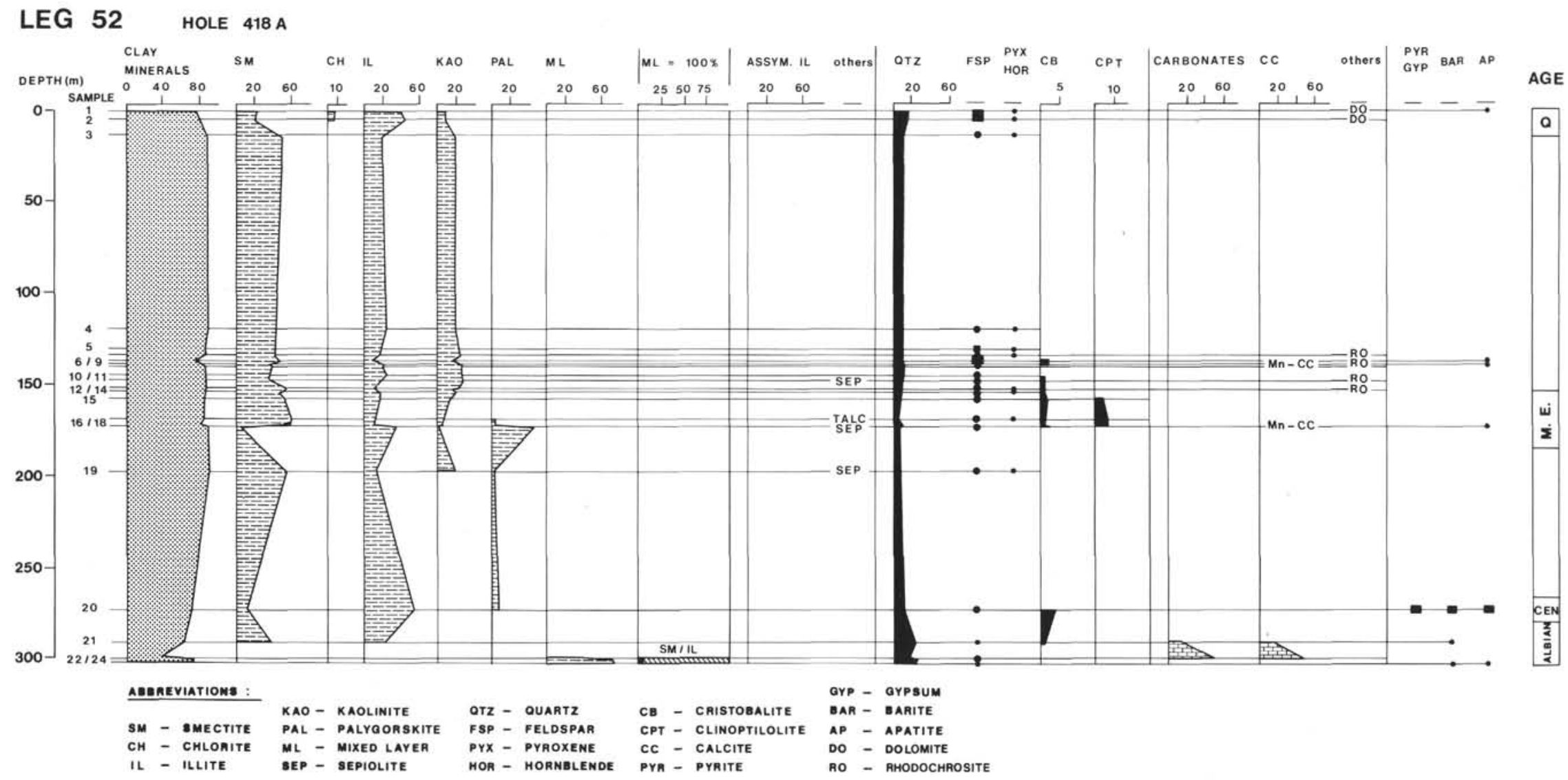

Figure 4. X-ray diffraction data, Hole $418 \mathrm{~A}$. 


\section{LEG 53 HOLE $418 \mathrm{~B}$}

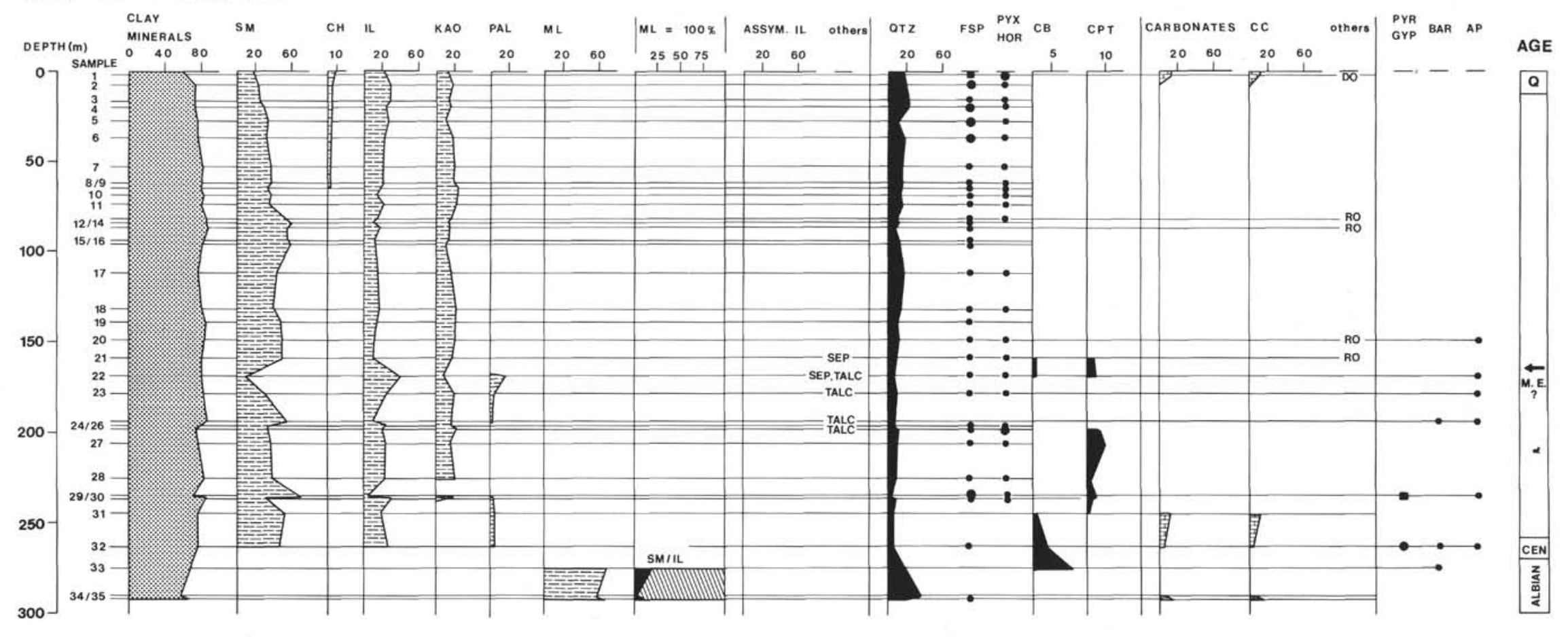

\section{AEmREVIATIONe :}

SM - MMECTITE KAO - KAOLINITE OTZ - QUARTZ

CM - CHLORITE ML - MIXED LAYER PYX - PYROXENE

IL - ILLITE SEP - SEPIOLITE HOR - MORNBLENDE

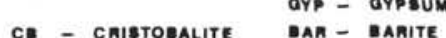

CPT - CLINOPTILOLITE AP - APATITE

Figure 5. X-ray diffraction data, Hole $418 B$.

$\begin{array}{ll}\text { CC - CALCITE } & \text { DO - DOLOMITE } \\ \text { PYR - PYRITE } & \text { RO }\end{array}$ 